\title{
The association between immune-related adverse events and survival outcomes in Asian patients with advanced melanoma receiving anti-PD-1 antibodies
}

Chiao-En Wu $\mathbf{u}^{1,2,3^{*}}$ (D), Chan-Keng Yang ${ }^{1,2,3}$, Meng-Ting Peng ${ }^{1,2,3}$, Pei-Wei Huang ${ }^{1,2,3}$, Ching-Fu Chang ${ }^{1,2,3}$, Kun-Yun Yeh ${ }^{3,4,5}$, Chun-Bing Chen ${ }^{3,5,6}$, Chih-Liang Wang ${ }^{3,5,7}$, Chao-Wei Hsu ${ }^{3,5,8}$, I-Wen Chen ${ }^{3,5,9}$, Cheng-Tao Lin ${ }^{3,5,10}$, Shir-Hwa Ueng ${ }^{3,5,11}$, Gigin Lin ${ }^{3,5,12}$, Yu-Fen Lin ${ }^{3,13}$, Chi-Yuan Cheng ${ }^{3,5,14}$ and John Wen-Cheng Chang 1,2,3*

\begin{abstract}
Background: The association between immune-related adverse events (irAEs) and survival outcomes in patients with advanced melanoma receiving therapy with immune checkpoint inhibitors (ICls) has not been well established, particularly in Asian melanoma.

Methods: We retrospectively reviewed 49 melanoma patients undergoing therapy with ICls (anti-PD-1 monotherapy), and analyzed the correlation between irAEs and clinical outcomes including progression-free survival (PFS) and overall survival (OS). Results: Overall, the patients who experienced grade 1-2 irAEs had longer PFS (median PFS, 4.6 vs. 2.5 months; HR, 0.52; 95\% Cl: 0.27-0.98; $p=0.042$ ) and OS (median OS, 15.2 vs. 5.7 months; HR, $0.50 ; 95 \% \mathrm{Cl}: 0.24-1.02 ; p=0.058$ ) than the patients who did not experience irAEs. Regarding the type of irAE, the patients with either skin/vitiligo or endocrine irAEs showed better PFS (median PFS, 6.1 vs. 2.7 months; HR, 0.40, 95\% Cl: 0.21-0.74; $p=0.003$ ) and OS (median OS, 18.7 vs. 4.5 months; HR, $0.34,95 \% \mathrm{Cl}: 0.17-0.69, p=0.003$ ) than patients without any of these irAEs.

Conclusions: Melanoma patients undergoing anti-PD-1 monotherapy and experiencing mild-to-moderate irAEs (grade 1-2), particularly skin (vitiligo)/endocrine irAEs had favorable survival outcomes. Therefore, the association between irAEs and the clinical outcomes in melanoma patients undergoing anti-PD-1 ICls may be severity and type dependent.
\end{abstract}

Keywords: Immune checkpoint inhibitors, irAE, Skin toxicity, Vitiligo, Endocrine, Melanoma, PD-1

\footnotetext{
*Correspondence: jiaoen@gmail.com; wen1902@hotmail.com

'Division of Haematology-Oncology, Department of Internal Medicine,

Chang Gung Memorial Hospital, Linkou, Taiwan

Full list of author information is available at the end of the article
}

(C) The Author(s). 2020 Open Access This article is licensed under a Creative Commons Attribution 4.0 International License, which permits use, sharing, adaptation, distribution and reproduction in any medium or format, as long as you give appropriate credit to the original author(s) and the source, provide a link to the Creative Commons licence, and indicate if changes were made. The images or other third party material in this article are included in the article's Creative Commons licence, unless indicated otherwise in a credit line to the material. If material is not included in the article's Creative Commons licence and your intended use is not permitted by statutory regulation or exceeds the permitted use, you will need to obtain permission directly from the copyright holder. To view a copy of this licence, visit http://creativecommons.org/licenses/by/4.0/. The Creative Commons Public Domain Dedication waiver (http://creativecommons.org/publicdomain/zero/1.0/) applies to the data made available in this article, unless otherwise stated in a credit line to the data. 


\section{Background}

Immune checkpoint inhibitors (ICIs), including ipilimumab, nivolumab, and pembrolizumab, have become standard therapies in advanced melanoma, regardless of genetic alteration [1-3]. The combination of ipilimumab and nivolumab demonstrated greater effectiveness than monotherapy with either of these [4-7]; however, combination therapy had the greater toxicity than monotherapy and insufficient power for overall survival (OS) over nivolumab limit the application of combination treatment in advanced melanoma. ICIs induce favorable response in some patients, so biomarkers are needed to determine the clinical course of ICIs in advanced melanoma.

One potentially effective clinical biomarker of ICI response in cancer patients is immune-related adverse event (irAE) [8]. Patients who experience irAEs during therapy with anti-PD-1 and anti-PD-L1 antibodies have been found to show favorable outcomes - overall response rate (ORR), progression-free survival (PFS), and OS - in cases of melanoma, lung cancer, and urothelial carcinoma [9-15]. However, as compared with other cancer types, the association between irAE occurrence and anti-PD-1 antibody efficacy is not well established in metastatic melanoma patients. Several retrospective studies have reported improved outcomes in patients who experience irAEs; however, not all measured outcomes consistently improved in these patients [16-19]. Besides, given most of the previous publications are studied on cutaneous melanoma, it raises the question that whether those results could be applied in Asian in which acral and mucosal melanoma are predominant.

Previously, we reported the clinical efficacy and safety of ICIs in our institution in 80 ICI-naïve melanoma patients [20]. As anti-CTLA-4, anti-PD-1 antibodies, and their combination showed distinct irAE patterns [2], we selected the patients undergoing anti-PD-l monotherapy, with either nivolumab or pembrolizumab, and analyzed the association between irAEs and survival outcomes. This study could provide additional evidence of irAEs as biomarkers of treatment outcomes.

\section{Methods}

\section{Patients}

All patients with histologically confirmed melanoma treated at the Chang Gung Memorial Hospital (CGMH), Linkou, during 2014 to 2019, were retrospectively reviewed. ICI-naïve patients undergoing anti-PD-1 antibody treatment were included in the current study. Only unresectable stage III and IV melanomas were included in current study. The patients with stage IV melanomas undergoing complete resection were excluded as ICI was used for adjuvant treatment in such cases. Patients who received other systemic treatments prior to ICI therapy, such as chemotherapy, targeted therapy, or cytokine therapy, were also included. A total of 49 ICI-naïve patients with advanced melanoma receiving anti-PD-1 antibodies, either nivolumab or pembrolizumab, were included in the study. The last follow-up timepoint included in the study was March 31, 2020.

\section{Treatment regimens and response evaluation}

The patients were treated with anti-PD-1 antibodies, either nivolumab ( $3 \mathrm{mg} / \mathrm{kg}$ every 2 weeks) or pembrolizumab $(2 \mathrm{mg} / \mathrm{kg}$ every 3 weeks), until disease progression or intolerable toxicity. The dose or schedule of anti-PD1 ICIs was adjusted by the physicians based on the patients' clinical condition and toxicity from treatment. Laboratory data on liver, renal, and endocrine function; cardiac enzymes; viral hepatitis status; and autoimmune antibodies were obtained before treatment and followed up regularly after starting treatment. Tumor response was evaluated by regular physical examination, chest radiography, computed tomography, or positron emission tomography.

\section{Patient characteristics and evaluation of outcomes}

Patient characteristics, including age, sex, Eastern Cooperative Oncology Group (ECOG) performance status, systemic treatment prior to ICIs, stage of melanoma, histologic types, and location of primary melanoma were recorded.

The irAEs were evaluated by a clinician based on the findings of laboratory tests, clinical examinations, and imaging studies. Cases with suspicious irAEs were discussed at meetings held by the Immuno-Oncology Center of Excellence of Chang Gung Memorial Hospital at Linkou, consisting of medical oncologists, pulmonologists, hepatologists, endoscopists, endocrinologists, dermatologists, neurologists, radiologists, nurses, etc. The irAEs (with a potential immunologic cause) were graded according to the National Cancer Institute Common Terminology Criteria for Adverse Events, version 4.0.

The principle of irAE management follows the clinical guideline and may be adjusted based on physicians' judgement and team discussion [21]. Generally, ICIs were withheld temporarily in the patients with mildmoderate irAE (grade 1-2) and systemic glucocorticoids / suppressants were not needed. Rechallenge of ICIs was applied when patients recovered from irAE. In contrast, systemic glucocorticoids/suppressants were applied in patients grade 3 and more irAE.

The RECIST (Response Evaluation Criteria in Solid Tumors) 1.1 Criteria were used to evaluate the best tumor response. PFS was defined as the length of time from the first day of ICI treatment until the first clinical or radiological evidence of disease progression, death, or latest follow-up timepoint. OS was defined as the length of time from the first day of ICI treatment until the date of death or last follow-up. 


\section{Statistical analysis}

The PFS and OS were estimated by the Kaplan-Meier method and compared by the log-rank test. Univariate analysis was performed to evaluate possible prognostic factors, and the results were presented as hazard radio (HR) and confidence interval (CI). IBM SPSS Statistics for Windows (Version 20.0, Armonk, NY, USA) was used for statistical analyses, where $P<0.05$ was considered statistically significant. This study was approved by the Institutional Review Board of CGMH (202000182B0). Patient consent to participate was not required because of the retrospective nature of this study, which was approved by the Institutional Review Board of CGMH.

\section{Results}

\section{Patient characteristics}

In the current study, a total of 49 advanced melanoma patients undergoing anti-PD-1 monotherapy (33 with nivolumab and 16 with pembrolizumab) as their firstline ICI treatment were included for retrospective analysis. Eighteen (36.7\%) received prior systemic treatments such as targeted therapy, cytokine therapy, or chemotherapy. The median age was 61 years. Twentytwo (44.9\%) were male and 27 (55.1\%) were female. Forty-two $(85.7 \%)$ patients had good performance status of $0-1$. Fifteen patients (30.6\%) had acral melanoma, 16 (32.7\%) had mucosal melanoma, and only five (10.2\%) had nonacral cutaneous melanoma. Six patients had locally advanced melanoma (stage III, 12.2\%), and 43 had metastatic melanoma (stage IV, 87.8\%). The patients' characteristics are summarized in Table 1 . The median PFS and median OS were 3.1 (95\% CI: 2.8-3.5) and 10.7 (95\% CI: 7.7-13.8) months, respectively (Supplementary Fig. S1). The ORR was $10.2 \%(n=5)$ including $2(4.1 \%)$ complete response.

\section{The pattern of irAE occurrence}

The median follow-up time was 9.1 months (range: 0.348.3 months). Overall, 30 (61.2\%) patients experienced irAEs of various etiologies. Three patients experienced severe irAE (grade 3-5) and one died of irAE of pneumonitis (grade 5). Another two patients had grade 3 pneumonitis and grade 3 hepatitis respectively. Skin lesions $(n=19,38.8 \%)$ was the most common irAE followed by endocrine irAE $(n=9,19.4 \%)$, fatigue $(n=7$, $14.3 \%)$, and colitis/diarrhea $(n=6,12.2 \%)$. The frequency of mucositis, vitiligo, liver- and lung-related irAEs were relatively low (<5\%) (Fig. 1a and Supplementary Table 1). In terms of vitiligo, some previous studies reported it is an irAE of interest in melanoma patients treated with ICIs and a possible predictor of favorable response and survival. However, only two patients had vitiligo in the current study so it was merged with other skin AEs for analyses (Figs. 1, 3, and Tables 2, 3).
Regarding the onset of irAEs, most skin-related irAEs occurred within the first month, but a few patients $(n=$ 2) experienced skin-related irAEs after 6 months of exposure to anti-PD-l antibodies. All endocrine-related irAEs occurred after 80-day exposure to anti-PD-1 antibodies. Fatigue occurred within the first 2 months. Two distinct patterns were found for diarrhea/colitis as half of the instances occurred within 1 month and the other half, after 3 months (Fig. 1b-c).

\section{The association between irAE and PFS}

Univariant analysis was performed to analyze the association between irAE and survival outcomes. Regarding overall irAEs, the patients who experienced grade 1-2 irAEs had significantly longer PFS than those who did not experience irAEs (median PFS, 4.6 vs. 2.5 months; HR, 0.52; 95\% CI: $0.27-0.98 ; p=0.042)$. In contrast, the patients who experienced severe irAEs (grade 3-5) had shorter PFS than those who did not experience any irAE (median PFS, 2.0 vs. 2.5 months; HR, 4.24; 95\% CI: 1.116.3; $p=0.035$ ) (Fig. 2a, Table 2).

Further, the patients with grade 1-2 skin/vitiligo (median PFS, 4.6 vs. 2.5 months; HR, 0.59; 95\% CI: 0.32-1.10; $p=0.096$ ) or grade $1-2$ endocrine irAEs (median PFS, 6.1 vs. 2.7 months; HR, 0.52; 95\% CI: $0.24-1.13 ; p=0.100$ ) showed favorable PFS without statistically significance (Supplementary Fig. S2). The patients with either irAEs had significantly better PFS than patients without skin/ vitiligo or endocrine irAEs (median PFS, 6.1 vs. 2.7 months; HR, 0.40; 95\% CI: 0.21-0.74, $p=0.003$ ) (Fig. 3a).

Unlike skin/vitiligo and endocrine irAEs, the patients with grade $1-2$ fatigue (median PFS, 2.4 vs. 3.2 months; HR, 2.56; 95\% CI: $1.09-6.00 ; p=0.030$ ) had significantly worse PFS than the patients without fatigue (Fig. 3c). In addition, the patients with lung-related irAEs had significantly worse PFS (median PFS, 0.9 vs. 3.2 months; HR, 6.71, 95\% CI: $1.44-31.38 ; p=0.016)$ than the patients without those irAEs (Fig. 3e). Of note, only two patients experienced lung-related irAEs and both had grade 3-5 irAEs, so the influence of grade 1-2 lung-related irAEs is unknown in the current study.

\section{The association between irAEs and OS}

Regarding the association between irAEs and OS, the patients who experienced grade 1-2 irAEs showed longer OS than the patients without irAEs (median OS, 15.2 vs. 5.7 months; HR, 0.50; 95\% CI: $0.24-1.02 ; p=0.058$ ). In contrast, the patents who experienced severe irAEs (grade 3-5) had shorter OS than the patients without any irAE (median OS, 2.0 vs. 5.7 months; HR, 5.13; 95\% CI: $1.29-20.34 ; p=0.020$ ) (Fig. 2b, Table 3). For different types of irAEs, the patients with grade 1-2 skin/vitiligo (median OS, 16.4 vs. 5.1 months; HR, 0.49; 95\% CI: $0.24-1.01 ; p=0.052)$ or grade $1-2$ endocrine irAEs 
Table 1 Patients' Characteristics

\begin{tabular}{|c|c|c|}
\hline Characteristics & Number & $\%$ \\
\hline \multicolumn{3}{|l|}{ Age (years) } \\
\hline Median (IQR) & $61(18)$ & \\
\hline$\leqq 60$ & 24 & 49.0 \\
\hline$>60$ & 25 & 51.0 \\
\hline \multicolumn{3}{|l|}{ Gender } \\
\hline Male & 22 & 44.9 \\
\hline Female & 27 & 55.1 \\
\hline \multicolumn{3}{|l|}{ Performance status } \\
\hline 0 & 29 & 59.2 \\
\hline 1 & 13 & 26.5 \\
\hline 2 & 6 & 12.2 \\
\hline 3 & 1 & 2.0 \\
\hline \multicolumn{3}{|l|}{ Location of primary site } \\
\hline Extremities & 18 & 36.7 \\
\hline Head and neck & 9 & 18.4 \\
\hline Trunk & 16 & 32.7 \\
\hline Unknown & 6 & 12.2 \\
\hline \multicolumn{3}{|l|}{ Type } \\
\hline Acral melanoma & 15 & 30.6 \\
\hline Non-acral cutaneous melanoma & 5 & 10.2 \\
\hline Mucosal melanoma & 16 & 32.7 \\
\hline Others & 7 & 14.3 \\
\hline Unknown & 6 & 12.2 \\
\hline \multicolumn{3}{|l|}{ Stage } \\
\hline 3 & 6 & 12.2 \\
\hline 4, M1a & 5 & 10.2 \\
\hline 4, M1b & 8 & 16.3 \\
\hline $4, \mathrm{M} 1 \mathrm{C}$ & 30 & 61.2 \\
\hline \multicolumn{3}{|l|}{ BRAF gene mutation } \\
\hline No & 34 & 69.4 \\
\hline Yes & 9 & 18.4 \\
\hline Unknown & 6 & 12.2 \\
\hline \multicolumn{3}{|l|}{ Treatment regimen } \\
\hline Nivolumab & 33 & 67.3 \\
\hline Pembrolizumab & 16 & 32.7 \\
\hline \multicolumn{3}{|l|}{ Immunotherapy therapy } \\
\hline First-line & 31 & 63.3 \\
\hline Second-or later-line & 18 & 36.7 \\
\hline \multicolumn{3}{|l|}{ Response } \\
\hline$C R$ & 2 & 4.1 \\
\hline$P R$ & 3 & 6.1 \\
\hline SD & 9 & 18.4 \\
\hline PD & 29 & 59.2 \\
\hline N/A & 6 & 12.2 \\
\hline
\end{tabular}

IQR interquartile range, $C R$ complete response, $P R$ partial response, $S D$ stable disease, $P D$ progressive disease, N/A not assessed (median OS, 18.7 vs. 8.8 months; HR, 0.38; 95\% CI: 0.24-1.13; $p=0.073$ ) showed favorable OS (Supplementary Fig. S2). On combining skin/vitiligo and endocrine irAEs, we found that the patients with either irAE had significantly better PFS than patients without these irAEs (median OS, 18.7 vs. 4.5 months; HR, 0.34; 95\% CI: $0.17-0.69, p=0.003$ ) (Fig. 3a).

Similar to the impact of fatigue in PFS, the patients with grade 1-2 fatigue (median OS, 3.7 vs. 11.3 months; HR, 2.69; 95\% CI: $1.14-6.34 ; p=0.024$ ) had significantly shorter OS than the patients without fatigue (Fig. 3d). In addition, the patients with lung-related irAEs had significantly shorter OS (median OS, 0.9 vs. 10.7 months; HR, 8.90; 95\% CI: $1.83-43.4 ; p=0.007)$ than the patients without lung-related irAEs (Fig. 3e).

\section{Discussion}

In the current study, the association between irAEs and clinical outcomes was investigated using data from 49 patients treated with anti-PD-1 antibodies in single institute cancer center; it was found that the association was dependent on type and severity of the irAEs. The patients with mild-to-moderate irAEs (grade 1-2) had better PFS and OS. In addition, patients with skin irAEs/ vitiligo/endocrine irAEs showed favorable PFS and OS.

Our findings were compatible with recently published meta-analysis investigating irAE and efficacy of ICIs and showing the occurrence of irAEs was significantly associated with a better ICI efficacy in cancer patients, particularly endocrine, skin, and low-grade irAEs [22]. In addition, this association was limited in PD-1 monotherapy but not anti-CTLA-4 nor combination therapy indicating different targets of ICIs might be associated with distinct irAE patterns which should be analyzed and discussed separately. Furthermore, ethnicity and melanoma subtype was found to be associated with distinct irAE profiling in the setting of PD-1 ICI by using 3 independent melanoma centers from the US. and China [23]. This finding indicates that not only melanoma subtype but also ethnicity influence the efficacy of anti-PD-1 ICI. NonCaucasian had higher rates of skin and endocrine irAEs but low rates of pneumonitis than Caucasian. Following previous findings, current study conducted in Taiwan provides additionally important and valuable evidence showing high frequency of skin/endocrine irAE and low frequency of pneumonitis were associated with survivals in Asian melanoma undergoing PD-1 melanoma.

The results of previous studies regarding to irAEs and survival in melanoma patients undergoing therapy with anti-PD-1 ICI have been inconsistent. Okada et al. examined 15 melanoma patients undergoing nivolumab therapy and found that patients with irAEs were associated with better OS than patients without irAEs [9]. Indini et al. conducted a retrospective analysis of 173 


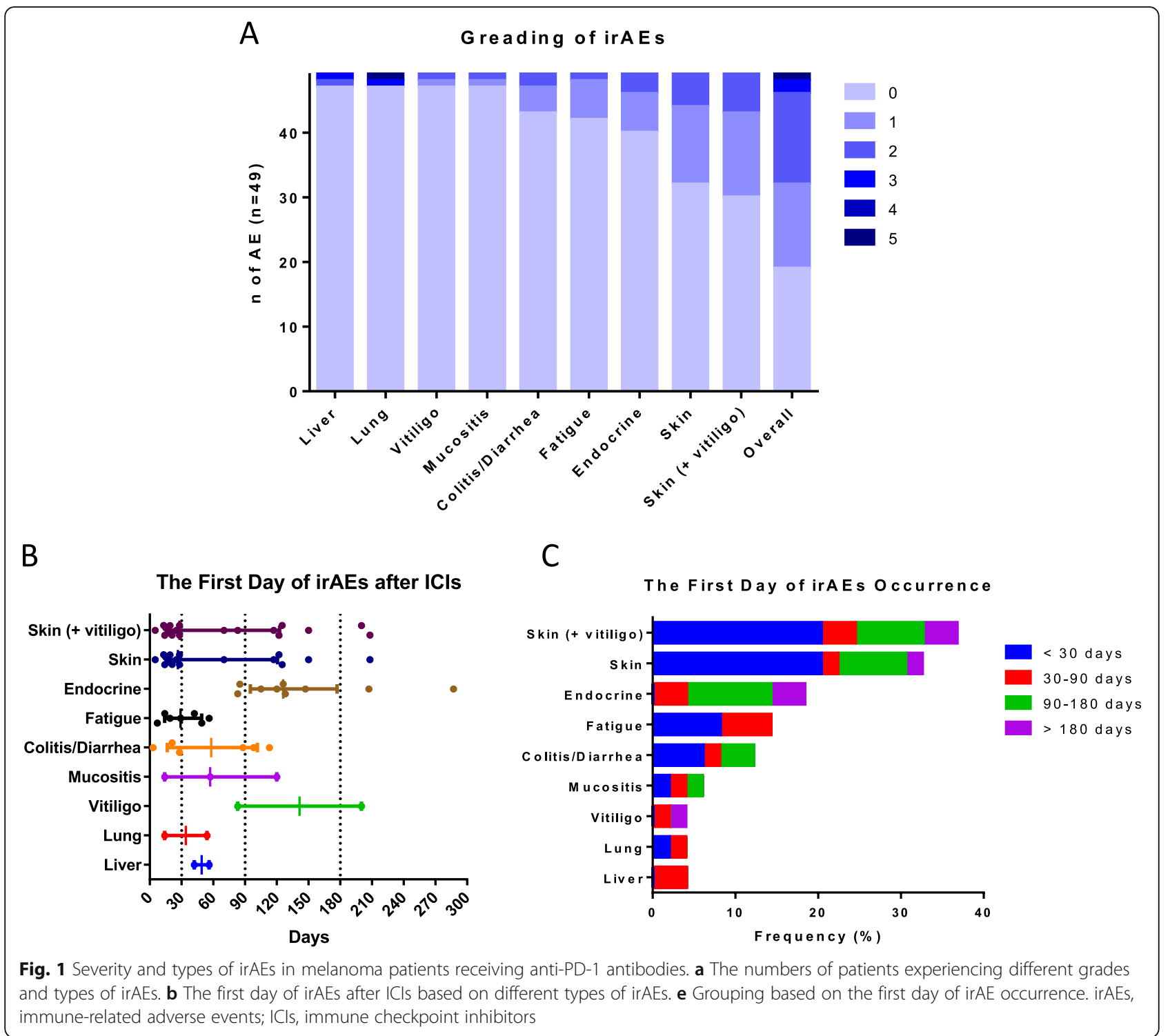

patients with metastatic melanoma treated with anti-PD-1 antibodies and found that $59 \%$ of the patients experiencing irAEs showed improved PFS and OS, independent of other factors [16]. However, a large retrospective study analyzed the outcomes of 576 melanoma patients pooled from several studies treated with nivolumab [18] and no differences in PFS were found between patients with or without irAEs. Our cohort showed distinct results-that the prognostic value of irAE was severity dependent.

In terms of severity of irAEs, mechanistically as irAEs are considered to be the bystander effects of activation of $\mathrm{T}$ cells by ICIs, so patients who experience more severe irAEs should have higher T-cell activity and experience better outcomes than those who experience mildto-moderate or no irAEs [24]. However, most of the previous studies on anti-PD- 1 and anti-CTLA- 4 antibodies rarely demonstrated the relationship between irAE severity and ICI efficacy. This variation in results could be attributed to the fact that patients experiencing severe irAEs tend to suffer from significant morbidity and sometimes mortality from the autoimmune reactions that compromise the benefit of ICIs [25]. In addition, severe toxicity is often associated with aggressive immunosuppression treatment, which may also influence the efficacy of ICIs [26]. Further, in the case of patients with severe irAEs, no ICIs were administered even after they completely recovered from the irAEs. Taken all together, these points could explain why the patients with severe irAEs had worse survival than patients without severe irAEs. One study in 858 older aged ( $\geq 65$ years) melanoma patients treated with ipilimumab supported our findings as patients with non-severe irAE had improved OS compared to patients without irAE, and patients with severe irAE appeared to have the highest risk of death 
Table 2 Univariate analysis of prognostic factors in progression-free survival

\begin{tabular}{|c|c|c|c|c|c|c|}
\hline Adverse events & Median (months) & 95\% C.I. of median & $P$ value & Hazard ratio & $95 \%$ C.I. of HR & $P$ value \\
\hline Skin & & & .274 & & & \\
\hline Grade $0(n=32)$ & 2.8 & $1.9-3.8$ & & 1 & & \\
\hline Grade $1-2(n=17)$ & 3.6 & $1.6-5.6$ & & 0.71 & $0.38-1.32$ & .278 \\
\hline Skin/vitiligo & & & .092 & & & \\
\hline Grade $0(n=30)$ & 2.5 & $1.6-3.4$ & & 1 & & \\
\hline Grade $1-2(n=19)$ & 4.6 & $2.5-6.7$ & & 0.59 & $0.32-1.10$ & .096 \\
\hline Mucositis & & & .287 & & & \\
\hline Grade $0(n=46)$ & 3.1 & $2.5-3.8$ & & 1 & & \\
\hline Grade $1-2(n=3)$ & 3.0 & $0.4-5.6$ & & 1.90 & $0.57-6.29$ & .296 \\
\hline Colitis & & & .624 & & & \\
\hline Grade $0(n=43)$ & 3.2 & $2.6-3.7$ & & 1 & & \\
\hline Grade $1-2(n=6)$ & 3.0 & $2.3-3.7$ & & 1.24 & $0.52-2.96$ & .626 \\
\hline Liver & & & .353 & & & \\
\hline Grade $0(n=47)$ & 3.2 & $2.7-3.6$ & & 1 & & \\
\hline Grade $1-2(n=1)$ & 3.1 & - & & 1.48 & $0.20-10.99$ & .700 \\
\hline Grade 3-5 $(n=1)$ & 2.1 & - & & 3.89 & $0.50-30.19$ & .194 \\
\hline Lung & & & .005 & & & \\
\hline Grade $0(n=47)$ & 3.2 & $2.8-3.5$ & & 1 & & \\
\hline Grade $3-5(n=2)$ & 0.9 & - & & 6.71 & $1.44-31.38$ & .016 \\
\hline Endocrine & & & .094 & & & \\
\hline Grade $0(n=40)$ & 2.7 & $2.1-3.3$ & & 1 & & \\
\hline Grade $1-2(n=9)$ & 6.1 & $2.7-9.6$ & & 0.52 & $0.24-1.13$ & .100 \\
\hline Fatigue & & & .024 & & & \\
\hline Grade $0(n=42)$ & 3.2 & $2.7-3.7$ & & 1 & & \\
\hline Grade $1-2(n=7)$ & 2.4 & $1.5-3.4$ & & 2.56 & $1.09-6.00$ & .030 \\
\hline Vitiligo & & & .234 & & & \\
\hline Grade $0(n=47)$ & 3.0 & $2.5-3.4$ & & 1 & & \\
\hline Grade $1-2(n=2)$ & 4.7 & - & & 0.32 & $0.04-2.33$ & .261 \\
\hline Skin/vitiligo/endocrine & & & .003 & & & \\
\hline Grade $0(n=26)$ & 2.3 & $1.7-2.8$ & & 1 & & \\
\hline Any grade $(n=23)$ & 4.8 & $4.3-5.3$ & & 0.40 & $0.21-0.74$ & .003 \\
\hline Overall & & & .001 & & & \\
\hline Grade $0(n=19)$ & 2.5 & $1.7-3.3$ & & 1 & & \\
\hline Grade $1-2(n=27)$ & 4.6 & $2.8-6.4$ & & 0.52 & $0.27-0.98$ & .042 \\
\hline Grade 3-5 $(n=3)$ & 2.0 & $0.2-3.8$ & & 4.24 & $1.1-16.3$ & .035 \\
\hline
\end{tabular}

C.I. confidence interval

[27], although ipilimumab rather than anti-PD-1 was used in this study.

In current study, we found that different types of irAEs differentially predicted survival, as skin/vitiligo/endocrine irAEs were favorable irAEs. Sanlorenzo et al. found that patients with cutaneous irAEs had a significantly longer PFS than those without in a retrospective analysis of 83 metastatic cancer patients (including 66 melanoma patients) treated with pembrolizumab [28]. Yamazaki et al. followed 124 Japanese melanoma patients treated with nivolumab and reported that the occurrence of skin-related and endocrine-related irAEs had a significant impact on the PFS of the patients [29] although only the abstract is available currently. Moreover, Fujisawa et al. demonstrated that endocrine-related irAEs were associated with longer OS of melanoma patients treated with ipilimumab after nivolumab [30]. Other than melanoma, a significant correlation between 
Table 3 Univariate analysis of prognostic factors in overall survival

\begin{tabular}{|c|c|c|c|c|c|c|}
\hline Adverse events & Median (months) & $95 \%$ C.I. of median & $P$ value & Hazard ratio & $95 \%$ C.I. of HR & $P$ value \\
\hline Skin & & & .054 & & & \\
\hline Grade $0(n=32)$ & 5.7 & $1.3-10.0$ & & 1 & & \\
\hline Grade $1-2(n=17)$ & 16.4 & $4.8-28.0$ & & 0.49 & $0.24-1.03$ & .059 \\
\hline Skin/vitiligo & & & .047 & & & \\
\hline Grade $0(n=30)$ & 5.1 & $3.1-7.2$ & & 1 & & \\
\hline Grade $1-2(n=19)$ & 16.4 & $4.8-28.0$ & & 0.49 & $0.24-1.01$ & .052 \\
\hline Mucositis & & & .150 & & & \\
\hline Grade $0(n=46)$ & 11.3 & $5.6-17.0$ & & 1 & & \\
\hline Grade $1-2(n=3)$ & 6.0 & $0.1-13.2$ & & 2.36 & $0.71-7.92$ & .163 \\
\hline Colitis & & & .773 & & & \\
\hline Grade $0(n=43)$ & 13.1 & $6.9-19.4$ & & 1 & & \\
\hline Grade $1-2(n=6)$ & 9.7 & 2.716 .6 & & 1.15 & $0.44-2.98$ & .773 \\
\hline Liver & & & .292 & & & \\
\hline Grade $0(n=47)$ & 10.7 & $5.3-16.2$ & & 1 & & \\
\hline Grade $1-2(n=1)$ & 9.7 & - & & 1.55 & $0.21-11.50$ & .670 \\
\hline Grade 3-5 $(n=1)$ & 2.8 & - & & 4.33 & $0.55-33.91$ & .162 \\
\hline Lung & & & .001 & & & \\
\hline Grade $0(n=47)$ & 10.7 & $6.2-15.3$ & & 1 & & \\
\hline Grade $3-5(n=2)$ & 0.9 & - & & 8.90 & $1.83-43.4$ & .007 \\
\hline Endocrine & & & .062 & & & \\
\hline Grade $0(n=40)$ & 8.8 & $2.4-15.3$ & & 1 & & \\
\hline Grade $1-2(n=9)$ & 18.7 & $2.0-35.3$ & & 0.38 & $0.13-1.09$ & .073 \\
\hline Fatigue & & & .019 & & & \\
\hline Grade $0(n=42)$ & 11.3 & $6.1-16.4$ & & 1 & & \\
\hline Grade $1-2(n=7)$ & 3.7 & $1.3-6.2$ & & 2.69 & $1.14-6.34$ & .024 \\
\hline Vitiligo & & & .856 & & & \\
\hline Grade $0(n=47)$ & 10.7 & $7.3-14.2$ & & 1 & & \\
\hline Grade $1-2(n=2)$ & 8.1 & - & & 0.83 & $0.11-6.16$ & .856 \\
\hline Skin/vitiligo/endocrine & & & .002 & & & \\
\hline Grade $0(n=26)$ & 4.5 & $0.9-8.1$ & & 1 & & \\
\hline Any grade $(n=23)$ & 18.7 & $6.2-31.1$ & & 0.34 & $0.17-0.69$ & .003 \\
\hline Overall & & & $<.001$ & & & \\
\hline Grade $0(n=19)$ & 5.7 & $0.1-11.7$ & & 1 & & \\
\hline Grade $1-2(n=27)$ & 15.2 & $7.9-22.5$ & & 0.50 & $0.24-1.02$ & .058 \\
\hline Grade 3-5 $(n=3)$ & 2.0 & $0.2-3.8$ & & 5.13 & $1.29-20.34$ & .020 \\
\hline
\end{tabular}

C.I. confidence interval

endocrine irAEs and OS was observed $(p=0.019)$ in a pooling analysis of 12 randomized controlled trials of 3815 metastatic head and neck and lung cancer patients treated with ICIs [31]. All of above studies supported our finding that skin and endocrine irAEs represent favorable irAEs.

Vitiligo is a specific irAE in melanoma and is considered a predictive biomarker of the effectiveness of ICIs in advanced melanoma as T cells activated by ICIs may recognize the common antigens on tumor cells and host melanocytes. Therefore, the occurrence of vitiligo reflects that the $\mathrm{T}$ cells are activated and ready to kill the melanomas [32]. In a retrospective report, Indini analyzed various irAEs and found that vitiligo was associated with better OS than other irAEs although not a statistically significant level $(p=0.061)$ [16]. Only two 


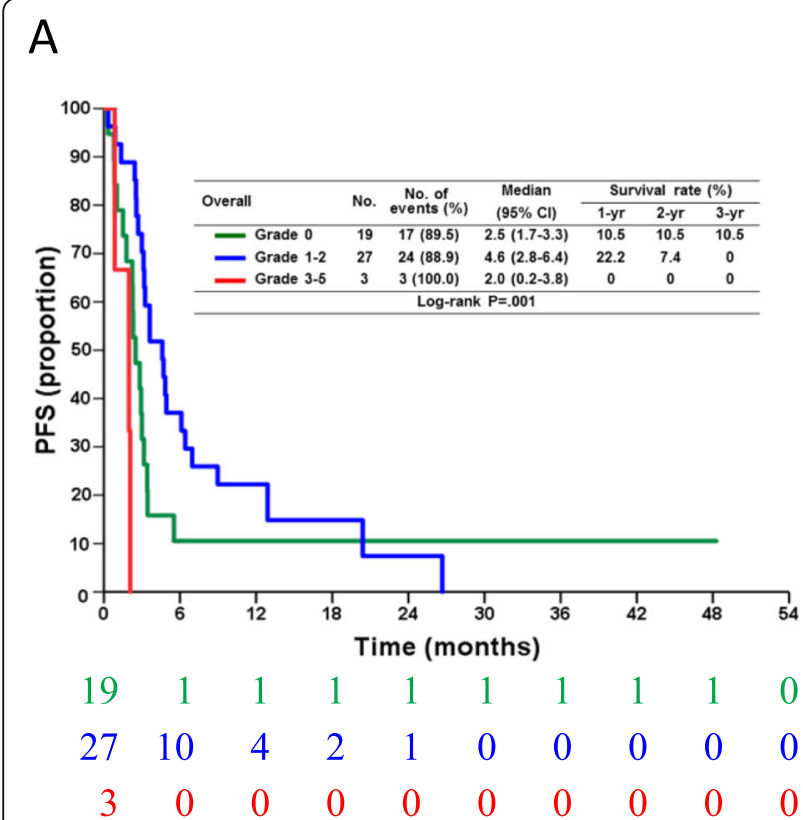

B

Fig. 2 Progression-free survival (PFS) (a) and overall survival (OS) (b) in melanoma patients undergoing anti-PD-1 antibodies based on severity of irAE The patients who experienced no irAEs, mild irAEs (grade 1-2), and severe irAEs (grade $3-5$ ) had significantly different PFS $(p=0.001)$ and OS $(p<0.001)$. The patients with mild irAEs showed favorable survival and those with severe irAEs showed unfavorable survival. The numbers below the charts correspond to patients at risk at each time point. irAEs, immune-related adverse events

patients experienced vitiligo in our cohort; therefore, the association between vitiligo and survival could not be undetermined.

Skin and endocrine irAEs in the current study were mild to moderate, so topical treatment and endocrine supplements were useful for managing the irAEs. Systemic steroids were not necessary for most of the patients who could continue ICI treatment. In contrast, pulmonary irAEs may be life threatening, so systemic steroids should be started as soon as pulmonary irAEs are suspected. The treatment course should be halted or terminated to improve the prognosis of patients with pulmonary irAEs. Fatigue is a nonspecific complaint resulting from ICI treatment, underlying malignancies or comorbidities. Therefore, patients with fatigue in our cohort may reflect disease progression rather than treatment-related AEs, which are difficult to differentiate in the initial presentation if patients have no other discomfort.

The association between irAEs and clinical outcomes was evident in not only metastatic melanoma but also resected melanoma with adjuvant therapy. In Keynote054, pembrolizumab was found to improve recurrencefree survival (RFS) of stage III melanoma patients after complete resection [33]. The occurrence of an irAE was associated with longer RFS in the pembrolizumab arm, particularly endocrine AE [34]. Although patients appeared to have a low risk of recurrence or death after vitiligo onset in the pembrolizumab arm, statistical significance was not observed due to the limited number of cases $(n=24)$. In addition, the severe irAEs (grade 34) were not significantly associated with favorable RFS, but the patients who experienced severe irAEs seemed to be numerically worse than those had not experienced severe irAE in the pembrolizumab arm. These findings are compatible with our findings on advanced melanoma as the severity and types of irAE predict the survival outcomes.

As the concern of glucocorticoids/immunosuppressant may suppress the efficacy of ICIs [35], withholding ICIs rather than glucocorticoids unless great $3 \mathrm{irAE}$ or pneumonitis [21]. The most frequent irAE in current study were skin and endocrine toxicities so topic glucocorticoids with or without oral antihistamine, and hormone replacement therapy were applied for those patients with skin and endocrine toxicities respectively. Only three patients experienced grade 3-5 irAE and two patients had pneumonitis, it is difficult to investigate the correlation and possible impact on survivals.

There are some limitations to the current retrospective study. Challenges of guarantee-time bias should be considered [36] because patients who experience irAEs are usually those who remain on ICI treatment for longer time periods and thus have a better prognosis than those who do not have irAEs. A retrospective study pooling melanoma patients from the randomized Checkmate 067 and Checkmate 069 trials suggested time in therapy is not the factor behind the relationship between irAE 


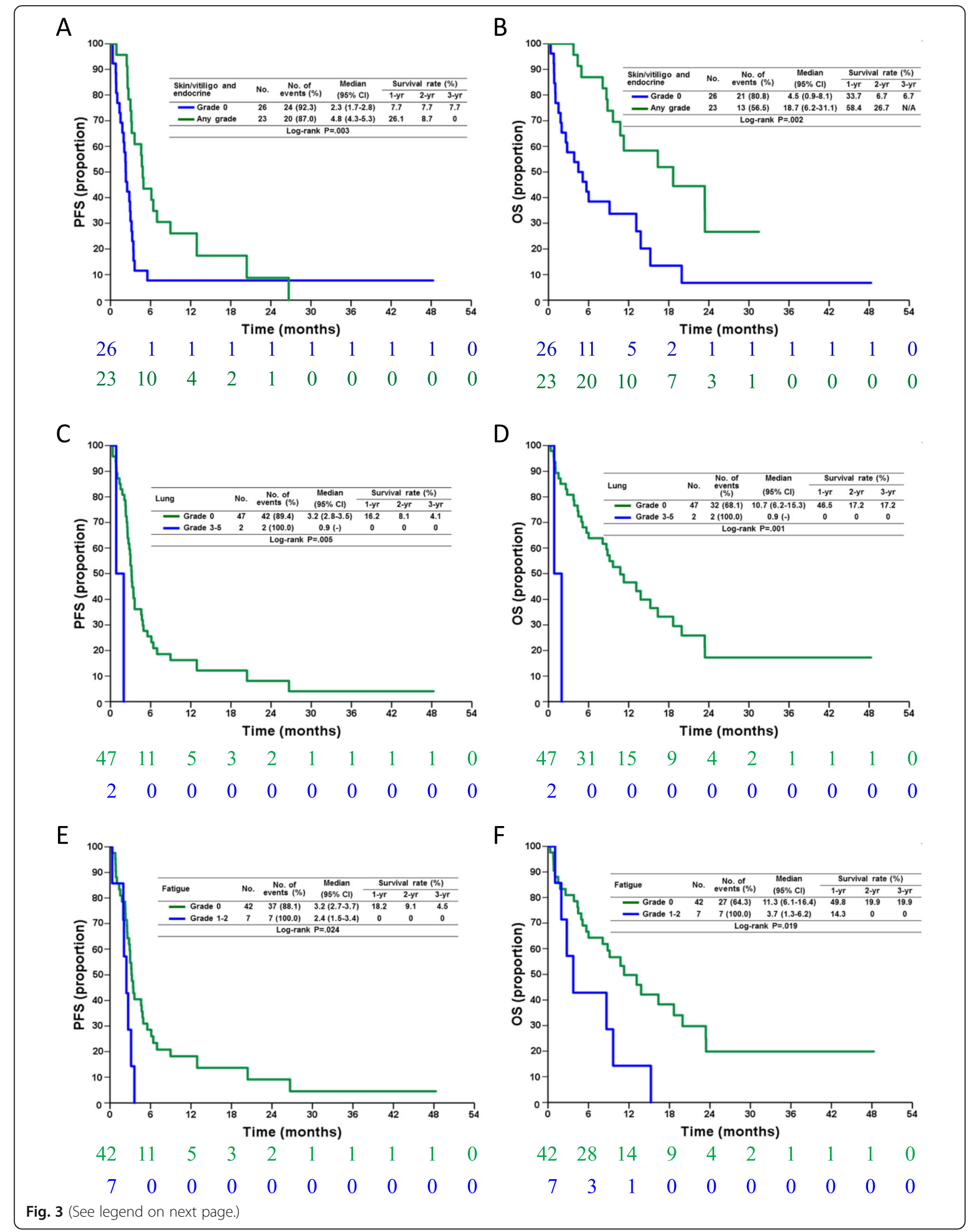


(See figure on previous page.)

Fig. 3 Progression-free survival (PFS) (a, $\mathbf{c}, \mathbf{e})$ and overall survival (OS) (b, d, $\mathbf{f})$ in melanoma patients receiving anti-PD-1 antibodies, who had skin/ vitiligo/endocrine $(\mathbf{a}, \mathbf{b})$, and lung $(\mathbf{c}, \mathbf{d})$ irAEs and fatigue $(\mathbf{e}, \mathbf{f})$. The patients experiencing skin/vitiligo/endocrine irAEs had favorable PFS and OS but those who experienced fatigue and lung irAEs had unfavorable PFS and OS. The numbers below the charts correspond to patients at risk at each time point. irAE, immune-related adverse events

onset and ICI efficacy [37]. A Cox model with a timevarying covariate was used to avoid this bias for KEYNOTE-054, and the hazard of recurrence or death was lower in the pembrolizumab-treated patients after irAE onset (HR 0.37; 95\% CI 0.24-0.57) than in those without or before irAE onset (HR 0.61; 95\% CI 0.49$0.77)(p=0.03)$ [34]. In current study, most skin irAE occurred in early period ( $<30$ days) of ICIs treatment indicating the early immune response of skin irAE may help clinician to predict the tumor response. In contrast, most endocrine irAE occurred after 3-month treatment so the prognostic value of endocrine irAE may be influenced by guarantee-time bias.

Small number of cases in current study limited the significance and some results should be interpreted cautiously. Only three patients experienced grade 3-5 irAE and two patients had pneumonitis so clinical significance of such irAE should not be confirmed using limited cases even statistical significance $(p<0.05$, Figs. 2,3$)$. The current study did not analyze the impact of autoimmune diseases as preexisting autoimmunity may be associated with ICIs efficacy and irAE [38]. However, the previous studies showed ICIs lead to similar rates of irAEs in patients with coexisting autoimmune diseases compared with those without existing coexisting autoimmune diseases [39].

\section{Conclusion}

In conclusion, melanoma patients undergoing anti-PD-1 ICIs who experienced mild-to-moderate irAE (grade 12) had favorable survival outcomes than those without irAE or severe irAE (grade 3-5). Patients with skin/vitiligo/endocrine irAEs had favorable survival. Therefore, the clinical outcomes of melanoma patients undergoing anti-PD-1 ICIs may be severity- and type-dependent, as per the current cohort.

\section{Supplementary information}

Supplementary information accompanies this paper at https://doi.org/10. 1186/s12885-020-07508-7.

Additional file 1: Supplementary Figure S1. Progression-free survival (PFS) and overall survival (OS) in melanoma patients receiving anti-PD-1 antibodies. The numbers below the charts correspond to patients at risk at each time point. Supplementary Figure S2. Progression-free survival (PFS) $(A, C, E)$ and overall survival (OS) (B, D, F) in melanoma patients receiving anti-PD-1 antibodies, who had skin $(A, B)$, vitiligo $(C, D)$, and endocrine $(E, F)$ irAEs. The patients experiencing skin/vitiligo $(C, p=0.092)$ and endocrine $(E, p=0.094)$ irAEs showed a trend of favorable PFS. The patients experiencing skin $(p=0.054)$, vitiligo $(p=0.047)$, and endocrine ( $p=$
0.062) irAEs had a trend of favorable OS. The numbers below the charts correspond to patients at risk at each time point. irAEs, immune-related adverse events.

Additional file 2: Supplementary Table 1. Adverse events.

\section{Abbreviations}

CGMH: Chang Gung Memorial Hospital; ECOG: Eastern Cooperative Oncology Group; HR: hazard radio; ICls: immune checkpoint inhibitors; irAE: immune-related adverse event; OS: overall survival; ORR: overall response rate; PFS: progression-free survival

\section{Acknowledgements}

This work was supported by Immuno-Oncology Center of Excellence, ChangGung Memorial Hospital at Linkou.

\section{Authors' contributions}

Study concept and design: CEW, CKY, MTP, PWH, CFC, KYY, CBC, CLW, CWH, IWC, CTL, SHU, GL, YFL, CYC, JWCC; Collection of data: CEW, CKY, MTP, PWH, CFC, JWCC; Analysis and interpretation of data: CEW, CKY, CFC; Drafting of the manuscript: CEW, CKY, MTP, PWH, CFC, KYY, JWCC; Critical revision of the manuscript for important intellectual content: CEW, CK, JWCC; Obtained funding: CEW, JWCC; Supervision: CEW, JWCC; All authors reviewed the results and approved the final version of the manuscript.

\section{Funding}

This work was supported by grants from Linkou Chang-Gung Memorial Hospital (CIRPG3H0061 2 and CORPG3J0151 2 to J.W-C.C.; CMRPG3I0451, CMRPG3J0971 3, CRRPG3K0021 2, CMRPG3K0601 and NMRPG3K6201 3 to C-E.W.). The funders had no role in the study design, data collection, analysis and interpretation, preparation of the manuscript and the decision to publish the results.

\section{Availability of data and materials}

The datasets generated AND analysed during the current study are not publicly available due to IRB regulation but are available from the corresponding author on reasonable request.

\section{Ethics approval and consent to participate}

This study was approved by the institutional review board (IRB) of CGMH (202000182BO). The consent to participate was not required because of retrospective entity of this study which was approved by IRB of CGMH.

\section{Consent for publication}

Not applicable.

\section{Competing interests}

None declared.

\section{Author details}

'Division of Haematology-Oncology, Department of Internal Medicine, Chang Gung Memorial Hospital, Linkou, Taiwan. ${ }^{2}$ Chang Gung University College of Medicine, 5, Fu-Hsing Street, Kwei-Shan, Taoyuan, Taiwan.

${ }^{3}$ Immuno-Oncology Center of Excellence, Chang Gung Memorial Hospital at Linkou, Taoyuan, Taiwan. ${ }^{4}$ Division of Hemato-Oncology, Department of Internal Medicine, Chang Gung Memorial Hospital, Keelung, Taiwan. ${ }^{5}$ Chang Gung University College of Medicine, Taoyuan, Taiwan. ${ }^{6}$ Department of Dermatology, Chang Gung Memorial Hospital at Linkou, Taipei, Keelung, Taiwan. ${ }^{7}$ Department of Thoracic Medicine, Chang Gung Memorial Hospital, Linkou, Taiwan. ${ }^{8}$ Department of Gastroenterology and Hepatology, Chang Gung Memorial Hospital, Linkou, Taiwan. ${ }^{9}$ Division of Endocrinology and Metabolism, Department of Internal Medicine, Chang Gung Memorial Hospital, Linkou, Taiwan. ${ }^{10}$ Department of Obstetrics and Gynecology, , 
Chang Gung Memorial Hospital, Linkou, Taiwan. ${ }^{11}$ Department of Pathology, Chang Gung Memorial Hospital, Linkou, Taiwan. ${ }^{12}$ Department of Medical Imaging \& Intervention, Chang Gung Memorial Hospital, Linkou, Taiwan. ${ }^{13}$ Department of Nursing, Chang Gung Memorial Hospital, Linkou, Taiwan.

${ }^{14}$ Department of Pharmacy, , Chang Gung Memorial Hospital, Linkou, Taiwan.

Received: 1 July 2020 Accepted: 7 October 2020

Published online: 21 October 2020

\section{References}

1. Hodi FS, O'Day SJ, McDermott DF, Weber RW, Sosman JA, Haanen JB, Gonzalez R, Robert C, Schadendorf D, Hassel JC, et al. Improved survival with ipilimumab in patients with metastatic melanoma. N Engl J Med. 2010; 363(8):711-23.

2. Hodi FS, Chiarion-Sileni V, Gonzalez R, Grob JJ, Rutkowski P, Cowey CL, Lao $C D$, Schadendorf D, Wagstaff J, Dummer R, et al. Nivolumab plus ipilimumab or nivolumab alone versus ipilimumab alone in advanced melanoma (CheckMate 067): 4-year outcomes of a multicentre, randomised, phase 3 trial. Lancet Oncol. 2018;19(11):1480-92.

3. Hamid O, Robert C, Daud A, Hodi FS, Hwu WJ, Kefford R, Wolchok JD, Hersey P, Joseph R, Weber JS, et al. Five-year survival outcomes for patients with advanced melanoma treated with pembrolizumab in KEYNOTE-001. Ann Oncol. 2019;30(4):582-8.

4. Larkin J, Chiarion-Sileni V, Gonzalez R, Grob JJ, Rutkowski P, Lao CD, Cowey $C L$, Schadendorf D, Wagstaff J, Dummer R, et al. Five-year survival with combined Nivolumab and Ipilimumab in advanced melanoma. N Engl J Med. 2019;381(16):1535-46.

5. Wolchok JD, Chiarion-Sileni V, Gonzalez R, Rutkowski P, Grob JJ, Cowey CL, Lao CD, Wagstaff J, Schadendorf D, Ferrucci PF, et al. Overall survival with combined Nivolumab and Ipilimumab in advanced melanoma. N Engl J Med. 2017;377(14):1345-56

6. Hodi FS, Chesney J, Pavlick AC, Robert C, Grossmann KF, McDermott DF, Linette GP, Meyer N, Giguere JK, Agarwala SS, et al. Combined nivolumab and ipilimumab versus ipilimumab alone in patients with advanced melanoma: 2-year overall survival outcomes in a multicentre, randomised, controlled, phase 2 trial. Lancet Oncol. 2016;17(11):1558-68.

7. Lebbe C, Meyer N, Mortier L, Marquez-Rodas I, Robert C, Rutkowski P, Menzies AM, Eigentler T, Ascierto PA, Smylie M, et al. Evaluation of two dosing regimens for Nivolumab in combination with Ipilimumab in patients with advanced melanoma: results from the phase IIIb/IV CheckMate 511 trial. J Clin Oncol. 2019;37(11):867-75.

8. Das $\mathrm{S}$, Johnson DB. Immune-related adverse events and anti-tumor efficacy of immune checkpoint inhibitors. J Immunother Cancer. 2019;7(1):306.

9. Okada N, Kawazoe H, Takechi K, Matsudate Y, Utsunomiya R, Zamami Y, Goda M, Imanishi M, Chuma M, Hidaka N, et al. Association between immune-related adverse events and clinical efficacy in patients with melanoma treated with Nivolumab: a multicenter retrospective study. Clin Ther. 2019;41(1):59-67.

10. Rogado J, Sanchez-Torres JM, Romero-Laorden N, Ballesteros Al, PachecoBarcia V, Ramos-Levi A, Arranz R, Lorenzo A, Gullon P, Donnay O, et al. Immune-related adverse events predict the therapeutic efficacy of anti-PD-1 antibodies in cancer patients. Eur J Cancer. 2019;109:21-7.

11. Riudavets M, Barba A, Maroto P, Sullivan IG, Anguera G, Paez D. Correlation between immune-related adverse events (irAEs) and efficacy in patients with solid tumors treated with immune-checkpoints inhibitors (ICls). J Clin Oncol. 2018;36(15). https://doi.org/10.1200/JCO.2018.36.15_suppl.3064.

12. Elias R, Yan F, Singla N, Levonyack N, Formella J, Christie A, Kapur P, Bowman Al, Hammers HJ, Hannan R, et al. Immune-related adverse events are associated with improved outcomes in $\mathrm{ICl}$-treated renal cell carcinoma patients. J Clin Oncol. 2019;37(7). https://doi.org/10.1200/JCO.2019.37.7_ suppl.645.

13. Grangeon M, Tomasini P, Chaleat S, Jeanson A, Souquet-Bressand M, Khobta N, Bermudez J, Trigui Y, Greillier L, Blanchon M, et al. Association between immune-related adverse events and efficacy of immune checkpoint inhibitors in non-small-cell lung Cancer. Clinical Lung Cancer. 2019;20(3): 201-7.

14. Sato K, Akamatsu H, Murakami E, Sasaki S, Kanai K, Hayata A, Tokudome N, Akamatsu K, Koh Y, Ueda H, et al. Correlation between immune-related adverse events and efficacy in non-small cell lung cancer treated with nivolumab. Lung Cancer. 2018;115:71-4.
15. Maher VE, Fernandes LL, Weinstock C, Tang SH, Agarwal S, Brave M, Ning YM, Singh H, Suzman D, Xu J et al: Analysis of the Association Between Adverse Events and Outcome in Patients Receiving a Programmed Death Protein 1 or Programmed Death Ligand 1 Antibody. J Clin Oncol 2019, 37(30):2730

16. Indini A, Di Guardo L, Cimminiello C, Prisciandaro M, Randon G, De Braud F, Del Vecchio M. Immune-related adverse events correlate with improved survival in patients undergoing anti-PD1 immunotherapy for metastatic melanoma. J Cancer Res Clin Oncol. 2019;145(2):511-21.

17. Borcoman E, Nandikolla A, Long G, Goel S, Le Tourneau C. Patterns of response and progression to immunotherapy. Am Soc Clin Oncol Educ Book. 2018;38:169-78.

18. Weber JS, Hodi FS, Wolchok JD, Topalian SL, Schadendorf D, Larkin J, Sznol M, Long GV, Li H, Waxman IM, et al. Safety profile of Nivolumab Monotherapy: a pooled analysis of patients with advanced melanoma. J Clin Oncol. 2017;35(7):785-92.

19. Suo A, Chan Y, Beaulieu C, Kong S, Cheung WY, Monzon JG, Smylie M, Walker J, Morris D, Cheng T. Anti-PD1-induced immune-related adverse events and survival outcomes in advanced melanoma. Oncologist. 2020; 25(5):438-46.

20. Wu CE, Yang CK, Peng MT, Huang PW, Lin YF, Cheng CY, Chang YY, Chen HW, Hsieh JJ, Chang JW. Immune Checkpoint Inhibitors for Advanced Melanoma: Experience at a Single Institution in Taiwan. Front Oncol. 2020. https://doi.org/10.3389/fonc.2020.00905.

21. Haanen J, Carbonnel F, Robert C, Kerr KM, Peters S, Larkin J, Jordan K, Committee EG: Management of toxicities from immunotherapy: ESMO Clinical Practice Guidelines for diagnosis, treatment and follow-up. Ann Oncol 2018, 29(Suppl 4):iv264-iv266.

22. Zhou $X$, Yao Z, Yang H, Liang N, Zhang $X$, Zhang F. Are immune-related adverse events associated with the efficacy of immune checkpoint inhibitors in patients with cancer? A systematic review and meta-analysis. BMC Med. 2020;18(1):87.

23. Bai X, Quach H, Cann CG, Zhang M, Kim MS, Kasumova GG, Si L, Tang B, Cui C, Yang X: Heterogeneous response and irAE patterns in advanced melanoma patients treated with anti-PD-1 monotherapy from different ethnic groups: Subtype distribution discrepancy and beyond. In.: American Society of Clinical Oncology; 2020.

24. Passat T, Touchefeu Y, Gervois N, Jarry A, Bossard C, Bennouna J. Physiopathological mechanisms of immune-related adverse events induced by anti-CTLA-4, anti-PD-1 and anti-PD-L1 antibodies in cancer treatment. B Cancer. 2018;105(11):1033-41.

25. Quach HT, Dewan AK, Davis EJ, Ancell KK, Fan R, Ye F, Johnson DB. Association of Anti-Programmed Cell Death 1 cutaneous toxic effects with outcomes in patients with advanced melanoma. Jama Oncol. 2019;5(6):906-8.

26. Arbour KC, Mezquita L, Long N, Rizvi H, Auclin E, Ni A, Martinez-Bernal G, Ferrara R, Lai W. Hendriks LEL, et al. Impact of baseline steroids on efficacy of programmed cell Death-1 and programmed death-ligand 1 blockade in patients with non-small-cell lung Cancer. J Clin Oncol. 2018;36(28): 2872-8.

27. Mian I, Yang M, Zhao H, Shah M, Diab A, Shannon V, Patel A, Amaria RN, Giordano SH, Suarez-Almazor ME: Immune-related adverse events and survival in elderly patients with melanoma treated with ipilimumab. J Clin Oncol. 2016;34(15). https://doi.org/10.1200/JCO.2016.34.15 suppl.3047.

28. Sanlorenzo M, Vujic I, Daud A, Algazi A, Gubens M, Luna SA, Lin K, Quaglino $P$, Rappersberger K, Ortiz-Urda S. Pembrolizumab cutaneous adverse events and their association with disease progression. Jama Dermatology. 2015; 151(11):1206-12.

29. Yamazaki N, Takahashi A, Namikawa K, Takenouchi T, Nakamura Y, Kitano S, Fujita T, Kubota K, Yamanaka T, Kawakami Y. Response of nivolumab monotherapy in 124 Japanese patients with advanced melanoma: interim analysis of prospective observational study (CREATIVE study). Ann Oncol. 2019;30.

30. Fujisawa Y, Yoshino K, Otsuka A, Funakoshi T, Uchi H, Fujimura T, Matsushita S, Hata H, Okuhira H, Tanaka R, et al. Retrospective study of advanced melanoma patients treated with ipilimumab after nivolumab: analysis of 60 Japanese patients. J Dermatol Sci. 2018;89(1):60-6.

31. Gomes-Lima CJ, Kwagvan J, King F, Fernandez SJ, Burman KD, Veytsman I: A comprehensive meta-analysis of endocrine immune-related adverse events of immune checkpoint inhibitors and outcomes in head and neck cancer and lung cancer. J Clin Oncol. 2019;37(15). https://doi.org/10.1200/JCO.2019. 37.15_suppl.e14096. 
32. Teulings HE, Limpens J, Jansen SN, Zwinderman AH, Reitsma JB, Spuls PI, Luiten RM. Vitiligo-like depigmentation in patients with stage III-IV melanoma receiving immunotherapy and its association with survival: a systematic review and meta-analysis. J Clin Oncol. 2015;33(7):773-81.

33. Eggermont AMM, Blank CU, Mandala M, Long GV, Atkinson V, Dalle S, Haydon A, Lichinitser M, Khattak A, Carlino MS, et al. Adjuvant

Pembrolizumab versus placebo in resected stage III melanoma. N Engl J Med. 2018;378(19):1789-801.

34. Eggermont AMM, Kicinski M, Blank CU, Mandala M, Long GV, Atkinson V, Dalle S, Haydon A, Khattak A, Carlino MS, et al. Association between immune-related adverse events and recurrence-free survival among patients with stage III melanoma randomized to receive Pembrolizumab or placebo: a secondary analysis of a randomized clinical trial. Jama Oncol. 2020

35. Ma Y, Yang H, Kroemer G. Endogenous and exogenous glucocorticoids abolish the efficacy of immune-dependent cancer therapies. Oncoimmunology. 2020;9(1):1673635.

36. Giobbie-Hurder A, Gelber RD, Regan MM. Challenges of guarantee-time bias. J Clin Oncol. 2013;31(23):2963-9.

37. Schadendorf D, Wolchok JD, Hodi FS, Chiarion-Sileni V, Gonzalez R, Rutkowski P, Grob JJ, Cowey CL, Lao CD, Chesney J et al: Efficacy and Safety Outcomes in Patients With Advanced Melanoma Who Discontinued Treatment With Nivolumab and Ipilimumab Because of Adverse Events: A Pooled Analysis of Randomized Phase II and III Trials. J Clin Oncol 2017, 35(34):3807.

38. Tahir SA, Gao J, Miura Y, Blando J, Tidwell RSS, Zhao H, Subudhi SK, Tawbi $H$, Keung E, Wargo J, et al. Autoimmune antibodies correlate with immune checkpoint therapy-induced toxicities. Proc Natl Acad Sci U S A. 2019; 116(44):22246-51.

39. Boland P, Pavlick AC, Weber J, Sandigursky S: Immunotherapy to treat malignancy in patients with pre-existing autoimmunity. J Immunother Cancer. 2020;8(1):e000356.

\section{Publisher's Note}

Springer Nature remains neutral with regard to jurisdictional claims in published maps and institutional affiliations.

Ready to submit your research? Choose BMC and benefit from:

- fast, convenient online submission

- thorough peer review by experienced researchers in your field

- rapid publication on acceptance

- support for research data, including large and complex data types

- gold Open Access which fosters wider collaboration and increased citations

- maximum visibility for your research: over $100 \mathrm{M}$ website views per year

At $\mathrm{BMC}$, research is always in progress.

Learn more biomedcentral.com/submissions 